\title{
Application of an in-situ Thermo-polymerized Porous Polymer: Creation of an On-column Frit for a Packed Capillary HPLC Column
}

\author{
Jiping MA, ${ }^{*}, * *$ Mingyu DING, ${ }^{* * \dagger}$ Yan Xu, ${ }^{* * *}$ and Lingxin CHEN** \\ *Institute of Environment \& Municipal Engineering, Qingdao Technological University, \\ Qingdao 266033, P. R. China \\ **Key Lab of Bioorganic Phosphorus Chemistry \& Chemical Biology, Ministry of Education, \\ Department of Chemistry, Tsinghua University, Beijing 100084, P. R. China \\ ***School of the Earth Sciences and Resources, China University of Geosciences, \\ Beijing 100083, P. R. China
}

\begin{abstract}
A 3-mm length of a porous monolithic polymer was prepared in a 0.32-mm inner-diameter fused-silica capillary by an insitu thermo-polymerization method and used as an on-column frit for a packed capillary HPLC column. The on-column frit can resist high pressure up to 400 bar. A 5- $\mu \mathrm{m}$ packing material was packed in the capillary with the on-column frit by a slurry method. At pressure driving mode, separation of samples was performed using the capillary HPLC column. The in-situ frit preparation method has the advantages of easy preparation, easy control of the location of the frit and a mild preparing reaction condition.
\end{abstract}

(Received August 22, 2005; Accepted October 30, 2006; Published March 10, 2007)

Capillary high-performance liquid chromatography (capillary HPLC), ${ }^{1-4}$ as an intermediate technique between conventional liquid chromatography and microchip separations, has been one of the main trends as a separation technique during the past decade. Especially, the development of proteomics and metabolomics has promoted the rapid development of capillary HPLC technology in recent years. Using capillary HPLC columns (typical size, $0.32-0.075 \mathrm{~mm}$ i.d.) has many advantages over using a common HPLC column (4.6 mm i.d.). Miniaturization of the separation column has led to lower running costs and reduced environmental pollution by solvents and chemicals. For example, a reduction of the column i.d. from 4.6 to $0.32 \mathrm{~mm}$ allows the same analysis with more than 200-times less solvent as the mobile phase. On the other hand, capillary HPLC has low sample-volume requirements and easy connection to mass spectrometry (MS). These advantages are paid more attention in the field of life sciences, since bioanalytical applications are often sample-limited. Nowadays, capillary HPLC coupled with MS as a complementary method for two dimensional polyacrylamide gel electrophoresis has become an important tool for separating protein..$^{5-7}$ However, the front-end column technology need to be further improved.

To date, there are mainly two kinds of capillary HPLC columns used. One is a packed column, typically filled with alkylated silica particles in fused-silica capillaries. The other is a monolithic column, ${ }^{8,9}$ which is prepared by in-situ polymerization and avoids frit fabrication. Although monolithic capillary columns are prepared much more easily than packed capillary columns, packed capillary columns are mainly employed in proteomics research because of the availability of

† To whom correspondence should be addressed.

E-mail: dingmy@chem.tsinghua.edu.cn; majiping2001@yahoo. com.cn various packing materials. However, the packing of micronsized particles into a capillary tubing and the preparation of solid and stable on-column frits at the end of the columns are difficult techniques.

Usually a capillary column needs tubing at the end of the column for connecting with the detector. The packed bed and the tubing for the detector are connected with a union or by glue. However, the connection can inevitably induce a dead volume. Considering the detrimental influence of the dead volume, the best design for the column is a zero dead volume connection between the packing bed and the tubing for the detector. If the packed bed and the tubing for the detector are integrated in one capillary, the detection window can be made very close to the frit, so as to obtain the minimum dead volume. In order to retain the packing material inside the fused-silica capillary, on-column frits have to be produced at the end of the packing bed.

Several frit-making methods have been developed. Sintering a silica packing material ${ }^{10}$ or together with sodium silicate ${ }^{11}$ has often been used for end-frit making. However, a partially intense heating in the sintering process can destroy the structure of particles, leading to some active sites on the surface of the frit, which causes permeability problems, resulting in capillary fragility at the frit location. The sintering process also involves uncontrollable temperature heating, and reproducibility of the frit-making is not easily obtained. In addition, some researchers have reported a frit-making method by a sol-gel technique at room temperature. ${ }^{12-14}$ However, the method requires extremely well-controlled conditions, since the sol-gel process leads to a dramatic shrinkage, which might lead to the creation of excessively large cavities within the large-bore capillary column. ${ }^{15}$ Chen et al. ${ }^{16}$ invented a method for preparing frits by photopolymerization. The frits are fabricated in fused-silica capillaries by the UV photopolymerization of a solution of 
glycidyl methacrylate and trimethylolpropane trimethacrylate. Although the polymerization frit-making technique has been used in CEC, this approach has not been investigated for a pressurized flow system.

The work presented here introduces a novel frit-making method. We prepare an on-column frit in a $0.32-\mathrm{mm}$ i.d. silicafused capillary by thermo-polymerization using a different polymer monomer from that mentioned above, butylmethacrylate (BMA) and an ethylenedimethacrylate (EDMA) polymerization solution. Compared with the on-column fritmaking method by UV photopolymerization, the capillary used in thermo-polymerization is more easily obtained. The packing material was packed in the capillary with an on-column frit by a slurry packing method. The capillary HPLC column has a zero dead-volume connection between the packing bed and the tubing for the detector. The frit can be permanently fixed in the capillary column by covalently bonding to the inner capillary surface, and it is capable of resisting at least up to 400 bar of packing pressure. In the pressure driving mode, the capillary HPLC column with on-column thermo-polymerization frit has been successfully applied to separate mixed samples. The porous structure, rigidity and permeability of the frit were also demonstrated in the present work.

\section{Experimental}

\section{Chemicals}

Methacrylic acid 3-(trimethoxysilyl)propyl ester was purchased from Tokyo Kasei Kogyo Co., Ltd. (Japan). Butylmethacrylate (BMA, 99\%) and ethylenedimethacrylate (EDMA, 99\%) were supplied by Acros Organics (USA). Azoisobutyronitrile (AIBN, analytical pure AR), thiourea (AR) were provided by Tianjin Fuchen Chemical Reagents Factory (China). Methanol was HPLC grade from Tianjin Kangde Science and Technology Ltd. (China). Propan-1-ol and 1,4butanediol (AR) were obtained from the Beijing Chemical Reagent Factory (China). Toluene (AR) was purchased from Atuozi Fine Chemical Engineering Co., Ltd. (China). Benzene, naphthalene, biphenyl, phenanthrene, anthracene (AR) were from the Beijing Shuanghua Fine Chemical Engineering Factory (China). Chloroform (AR) was from Beijing Modern Chemical Ltd. (China). Deionized water used was all redistilled.

\section{Instrumentation and materials}

A fused-silica capillary (0.32 mm i.d., $0.45 \mathrm{~mm}$ o.d.) was purchased from Reafine Chromatography Ltd. (Hebei, China). The packing material, $5 \mu \mathrm{m}$ Chromatorex SMB-300-C18, was obtained from Fuji (Japan). A self-made slurry reservoir was 5 $\mathrm{mm}$ i.d. $\times 26 \mathrm{~mm}$ length made of stainless-steel. A scanning electron microscope (SEM) Model KYKY 2800 (Chinese Academy of Sciences, Scientific Instrument Research and Manufacture Center, China) was used. A mercury potosimeter AutoPore IV 9510 (Micromeritics Inc., USA) was used for determining of the pore-size distribution of the porous polymer.

An Alltech 426 HPLC pump was used to evaluate the column performance. A six-port injection valve with a $200 \mathrm{nl}$ loop was used. A T union was used to split delivering appropriate amounts of the mobile phase. The capillary column inlet was installed in the injection valve using a PEEK sleeve $(0.5 \mathrm{~mm}$ i.d., $1.6 \mathrm{~mm}$ o.d.) and a screwed joint. On-column detection was carried out using a CL2001 changeable wavelength ultraviolet-visible absorbance detector (Beijing Cailu Scientific Instrument Ltd., China) with a modification. Chromatograms
Table 1 Polymerization mixture filled in a capillary for fritmaking

\begin{tabular}{llc}
\hline & \multicolumn{1}{c}{ Compound } & Ratio \\
\hline \multirow{2}{*}{ Monomer mixture (w/w) } & BMA & 59.5 \\
& EDMA & 39.5 \\
& AIBN & 1 \\
Porogen mixture (w/w) & 1-Propanol & 50 \\
& 1,4-Butanediol & 40 \\
& Water & 10 \\
Polymerization mixture (v/v) & Monomer mixture & 40 \\
& Porogen mixture & 60
\end{tabular}

were recorded using the computer software N2000 chromatography data system supplied by Zhida Information Engineering Ltd., Zhejiang University, China.

\section{Frit preparation}

In-situ thermo-polymerization method. Because the frit must be firmly bound to the capillary inner wall, the capillary was pretreated prior to the polymerization reaction. We used the bifunctional solvent methacrylic acid 3-(trimethoxysilyl)propyl ester, which was often used to silanize the capillary wall, and at the same time provided a functional group to covalently bind with a methacrylate ester polymerization mixture. ${ }^{17}$ A $0.32-\mathrm{mm}$ i.d. fused-silica capillary was flushed with $0.1 \mathrm{M}$ sodium hydroxide for $2 \mathrm{~h}$ to activate the capillary inner surface and to convert its siloxane groups into silanol ones. The capillary was then flushed with water untill the water flowing from the capillary was tested as neutral, and flushed with a 50-times capillary volume of acetone. The capillary was dried by the passage of nitrogen for $5 \mathrm{~min}$. The capillary was filled with a silanization solution containing $30 \%(\mathrm{v} / \mathrm{v})$ methacrylic acid 3(trimethoxysilyl)propyl ester dissolved in acetone, sealed with septa and then kept at room temperature for $12 \mathrm{~h}$. After silanization, the capillary was flushed with 50-times capillary volume of acetone, and dried by the passage of nitrogen for 5 $\min$.

In the next step, the capillary was filled with a suitable ratio of a polymerization mixture solution. According to Refs. 18 and 19 , the polymerization solution consisted of a monomer mixture (containing functional monomer BMA, crosslinking agent EDMA and initiator AIBN) and a porogen solvent (propan-1-ol, 1,4-butanediol and deionized water). Table 1 shows the suitable ratio of the polymerization solution for frit-making. Firstly, the capillary was filled with the polymerization solution; then, the two ends of the capillary were sealed with a silicon rubber septa. Finally, the capillary was thermostatted at $60^{\circ} \mathrm{C}$ for $20 \mathrm{~h}$. After polymerization, the capillary was connected to the injection valve and flushed with methanol.

Sintering silica packing material method. A frit at one end of fused-silica capillary column was fabricated using a sodium silicate paste mixed with $2 \mu \mathrm{m}$ porous silica particles (Dalian Institute of Chemical Physics, Chinese Academy of Sciences, China) and heated in an automatic time-programmed oven (the oven was time-controllable, ranging from a few seconds to several minutes, laboratory-made) at about $300^{\circ} \mathrm{C}$ for about $10 \mathrm{s.} .^{20}$ Preparation of the capillary HPLC. The $0.32-\mathrm{mm}$ i.d. of a fused-silica capillary with a frit made by in-situ polymerization, as shown above, was slurry packed with a reversed-phase packing material. The slurry packing apparatus consisted of a pump, an ultrasonic bath and a slurry reservoir. One end of the capillary was connected to the slurry reservoir by means of a screwed joint and a piece of $1.6 \mathrm{~mm}$ o.d. PEEK tubing as a 

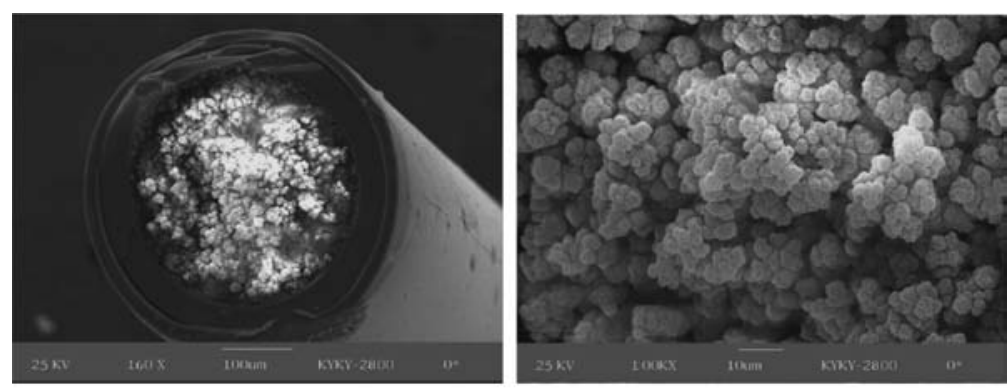

Fig. 1 Scanning electron micrograms of a frit made by in situ polymerization.

sleeve. The slurry was prepared with $0.2 \mathrm{~g}$ of a $5-\mu \mathrm{m}$ ODS in $0.5 \mathrm{ml}$ of chloroform and treated in an ultrasonic bath for $5 \mathrm{~min}$. The slurry was then transferred to the packing reservoir. The column was filled by using a constant flow until a final pressure of 400 bar was reached. This pressure was then maintained for several hours. The flow-rate was gradually decreased to make the pressure reduced to zero. When the pressure was decreased to zero, the capillary column was disconnected with the slurry reservoir. The whole column was about $32 \mathrm{~cm}$ long and the packing bed was $20 \mathrm{~cm}$ long. An on-column detection window was made close to the frit at a packing-free part of the column by scraping off the capillary polyimide coating, which was brought into the light path of an UV absorbance detector.

\section{Results and Discussion}

\section{Thermo-polymerization conditions}

To prepare a good frit, the condition of the polymerization should be investigated. A good frit should have suitable permeability and rigidity. The permeability of the frit is related to the porosity. A larger porosity means a higher permeability. On the other hand, a larger porosity leads to worse rigidity. Some variables affecting the polymerization and the properties of the monolithic material have already been studied for monolithic column preparation in HPLC systems and CEC systems, such as the temperature, radical reaction time, composition and percentage of the porogenic agents in the polymerization mixture, and content of the cross-linker and initiator. ${ }^{21,22}$

The higher is the polymerization temperature, the smaller are the pores obtained. The use of shorter reaction times leads to produce larger pores. Increasing the proportion of the crosslinker in the monomer mixture leads to a decrease in average pore size. When the content of the porogen solvent in the polymerization mixture is reduced, the porosity decreases. A higher concentration of the initiator leads to smaller pores. The porosity is sensitive to the amount of propan-1-ol. As the content of the propan-1-ol in porogen solvent increases, the porosity decreases. When we used the same BMA:EDMA ratio (59.5:39.5\%, w/w) and 1\% (w/w) of AIBN was kept constant, the content of the porogen solvent in the polymerization mixture was changed from 60 to $70 \%$, and the content of the propan-1-ol in porogen solvent was from 30 to $50 \%$; the frits showed acceptable permeability and rigidity.

\section{Characteristics of the frit}

Different lengths of frits were made from 1 to $6 \mathrm{~mm}$. The experimented results showed that a 1-mm length of the frit could not endure a high pressure and was washed out of the capillary. A longer frit, such as 5-6 mm length, had a higher

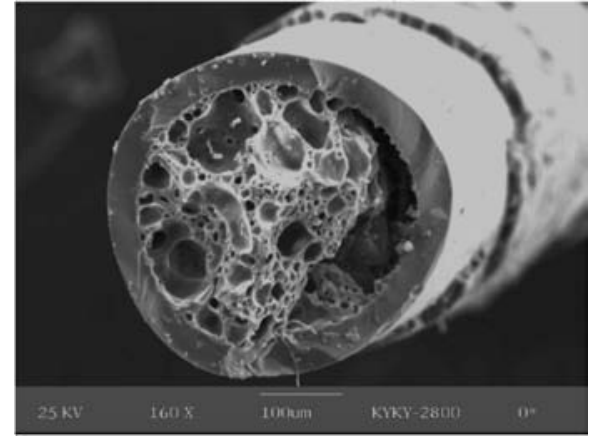

Fig. 2 Scanning electron micrograms of a frit made by the sintering silica packing material method.

resistance than the shorter one; also, the long frit had a potential of affecting the separation performance of the capillary HPLC column. Based on the above consideration, one frit with $3 \mathrm{~mm}$ length was chosen. To obtain a frit with good rigidity, we pretreated the inner wall of the capillary, and investigated the ratio of the polymerization solvent. Another consideration is the choice of the fused-silica capillary. A $0.53-\mathrm{mm}$ i.d. of capillary with a common wall thickness was not very suitable for making the capillary HPLC column, since it can not endure a higher pressure than a smaller i.d. of the capillaries. However, if we choose a specified thicker wall of the capillary, the fritmaking method described in this paper is also applicable. On the other hand, the frit in much thinner capillaries (such as 0.05 $\mathrm{mm}$ i.d.) can not be easily prepared. In the typical innerdiameter range of capillary columns for micro-HPLC $(0.10-$ $0.32 \mathrm{~mm}$ i.d.), this method can be used. As a demonstration, we made the frit in a capillary of $0.32 \mathrm{~mm}$ i.d. The results showed that the frit provided a high mechanical strength, sufficient for a packing pressure up to 400 bar for a 3-mm length frit on a $0.32-\mathrm{mm}$ i.d. fused-silica capillary.

The permeability of the frit is acceptable, since the back pressure is 2 bar at a flow-rate of $10 \mu \mathrm{min}^{-1}$ with methanol used as the mobile phase. Figure 1 shows a photo of the frit produced by SEM. The frit exhibits a porous appearance. Figure 2 shows an SEM picture of a frit made by the sintering silica packing material method. From Figs. 1 and 2, we can see that the sintering silica packing material frit did not have a uniform pore distribution as an in-situ polymerization frit. Moreover, the polyimide coating outside of the fused silica capillary was broken when frit-making by the sintering silica packing material method, which may lead to fragility of the capillary. The average pore size of the frit was $6.1 \mu \mathrm{m}$, as determined using a mercury intrusion porosimeter. Although the packing material size was smaller than several pores size of 


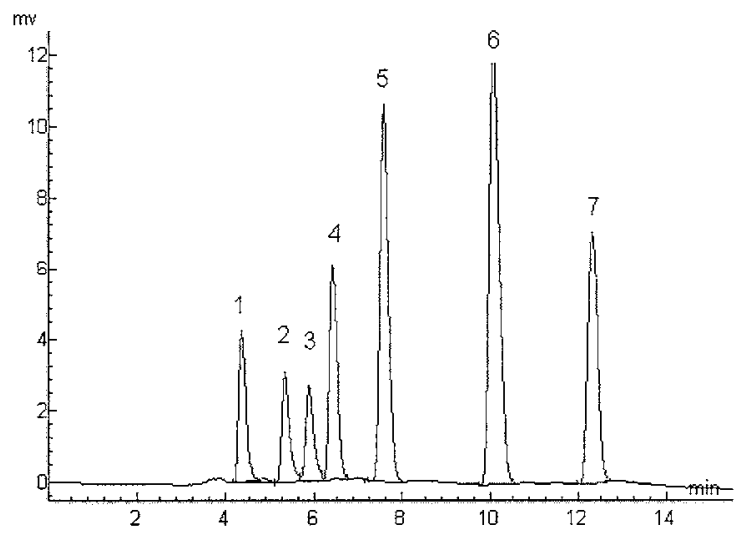

Fig. 3 Capillary HPLC separation of seven components on a reversed-phase column with an in situ polymerization frit. Conditions: $20 \mathrm{~cm} \times 0.32 \mathrm{~mm}$ i.d. column packed with ODS $5 \mu \mathrm{m}$ particles. Isocratic elution, methanol/water (75:25); column flow-rate, $3 \mu \mathrm{l}$ $\mathrm{min}^{-1}$; UV on-column detection at $254 \mathrm{~nm}$; injection volume, $200 \mathrm{nl}$. Peaks: 1, thiourea; 2, benzene; 3, toluene; 4, naphthalene; 5, biphenyl; 6 , phenanthrene; 7 , anthracene.

the frit, no particles of the packing were found to pass through the frit pores during either the packing procedure or capillary HPLC applications. This was because of cooperative effects of the tortuous paths of the pores and the presence of bottleneckshaped pores within the monolithic structure.

The total porosity $\left(\varepsilon_{\mathrm{T}}\right)$ is an important parameter for frit evaluation. It is rather difficult to measure the porosity for a 3 $\mathrm{mm}$ frit. Therefore, frits of $150 \mathrm{~mm}$ long were made, and the porosity was measured. In this study, the flow method was used to measure the $\varepsilon_{\mathrm{T}}$ values. The calculation of $\varepsilon_{\mathrm{T}}$ was based on the equation $\varepsilon_{\mathrm{T}}=4 F t_{0} /\left(d^{2} \pi L\right)$, where $F$ is the volumetric flow-rate, $t_{0}$ the retention time of an unretained marker (thiourea), $d$ the column inner diameter, and $L$ the column length. A flow-rate of $1 \mu \mathrm{l} \mathrm{min}-1$ was applied for $\varepsilon_{\mathrm{T}}$ measurements. The value of $\varepsilon_{\mathrm{T}}$ for the in-situ thermo-polymerization frit was calculated to be 0.66 . Seven frits were made by the method; the RSD of porosity was $5 \%$.

\section{Column application}

In the capillary HPLC system, on-column UV detection is commonly used due to its convenience and overall robustness. However, its main disadvantage is the limited concentration sensitivity, which is a function of the flow cell path length. If high sensitivity is required, mass spectrometry can be chosen to obtain significant gains in sensitivity. Since this research focuses on frit preparation for a capillary HPLC column, the sensitivity is not very important. We chose on-column UV detection in this work. A standard mixture sample was used to test the separation performance of the capillary column with an in-situ thermo-polymerization frit (Fig. 3). Although the chromatogram shown here does not have very high separation efficiencies, the structure of the capillary column presented in this paper determines that it can minimize the dead volume, and thus the column should produce a higher column efficiency if it is better packed. The frit was sufficiently stable enough to safely hold back the packing bed, and no bleeding of the particles was observed. It was proved that the frit was stable and effective in at least 50 repeated operations. Since the aim of this work was to demonstrate that the frit made by this thermo-polymerization method can withstand high pressure and retain the packing material inside the fused-silica capillary, the above experimental results are satisfactory.

\section{Conclusions}

This paper describes another application of an in-situ thermopolymerized porous polymer, that is, making an on-column frit for a capillary HPLC column. The results prove that it is a suitable method to produce the on-column frit in fused-silica columns. The column with the frit showed good permeability and rigidity when it was operated in the pressure-driven flow mode. The fabrication of monolithic frits using thermopolymerization has many advantages, such as a simple preparation process, and fine control of the porous properties as well as the location of the frits.

\section{References}

1. C. G. Horváth, B. A. Preiss, and S. R. Lipsky, Anal. Chem., 1967, 39, 1422.

2. D. Ishii, K. Asai, K. Hibi, T. Jonokuchi, and M. Nagaya, J. Chromatogr., 1977, 144, 157.

3. J. P. C. Vissers, J. Chromatogr., A, 1999, 856, 117.

4. L. X. Chen, Y. F. Guan, and J. P. Ma, Prog. Chem., 2003, $15,107$.

5. D. B. Kassel, B. Sushan, T. Sakuma, and J. P. Salzmann, Anal. Chem., 1994, 66, 236.

6. J. R. Yaters, J. K. Eng, and A. L. Mcormack, Anal. Chem., 1995, 67, 3202.

7. S. Hsien, K. Dreisewerd, R. C. Vanderschors, C. R. Jimenez, J. Z. Stall, F. Hillenkamp, J. W. Jorgenson, W. P. Ceraerts, and K. W. Li, Anal. Chem., 1998, 70, 1847.

8. S. Hjerten, J. L. Liao, and R. Zhang, J. Chromatogr., 1989, 473, 273.

9. F. Svec and J. M. J. Frechet, Anal. Chem., 1992, 64, 820.

10. R. Asiaie, X. Huang, D. Farnan, and C. Horvath, J. Chromatogr., A, 1998, 806, 251.

11. Y. Chen, G. Gerhardt, and R. Cassidy, Anal. Chem., 2000, 72,610 .

12. M. T. Dulay, R. P. Kulkarni, and R. N. Zare, Anal. Chem., 1998, 70, 5103.

13. M. Schmid, F. Bauml, A. P. Kohne, and T. Welsch, J. High Resol. Chromatogr., 1999, 22, 438.

14. X. M. Zhang and S. Huang, J. Chromatogr., A, 2001, 910, 13.

15. J. R. Chen, R. N. Zare, E. C. Peters, F. Svec, and J. M. J. Frechet, Anal. Chem., 2001, 73, 1987.

16. J. R. Chen, M. T. Dulay, R. N. Zare, F. Svec, and E. C. Peters, Anal. Chem., 2000, 72, 1224.

17. Ch. Ericson, J. L. Lio, K. Nakazato, and S. Hjerten, J. Chromatogr., A, 1997, 767, 33.

18. P. Coufal, M. Cihak, J. Suchankova, E. Tesarova, Z. Bosakova, and K. Stulık, J. Chromatogr., A, 2002, 946, 99.

19. X. Shu, L. X. Chen, B. C. Yang, and Y. F. Guan, J. Chromatogr., A, 2004, 1052, 205.

20. L. X. Chen, Y. F. Guan, J. P. Ma, G. A. Luo, and K. H. Liu, J. Chromatogr., A, 2005, 1064, 19.

21. F. Svec, E. C. Peters, D. Sykora, and J. M. J. Frechet, J. Chromatogr., A, 2000, 887, 3.

22. H. Zou, X. Huang, M. Ye, and Q. Luo, J. Chromatogr., A, $\mathbf{2 0 0 2}, 954,5$. 